\title{
Interface trap characterization and electrical properties of Au-ZnO nanorod Schottky diodes by conductance and capacitance methods
}

I Hussain, Muhammad Yousuf Soomro, Nargis Bano, Omer Nur and Magnus Willander

\section{Linköping University Post Print}

N.B.: When citing this work, cite the original article.

Original Publication:

I Hussain, Muhammad Yousuf Soomro, Nargis Bano, Omer Nur and Magnus Willander, Interface trap characterization and electrical properties of $\mathrm{Au}-\mathrm{ZnO}$ nanorod Schottky diodes by conductance and capacitance methods, 2012, Journal of Applied Physics, (112), 6, 064506. http://dx.doi.org/10.1063/1.4752402

Copyright: American Institute of Physics (AIP) http://www.aip.org/

Postprint available at: Linköping University Electronic Press http://urn.kb.se/resolve?urn=urn:nbn:se:liu:diva-85203 


\title{
Interface trap characterization and electrical properties of Au-ZnO nanorod Schottky diodes by conductance and capacitance methods
}

\author{
I. Hussain, M. Y. Soomro, N. Bano, O. Nur, and M. Willander \\ Department of Science and Technology, Campus Norrköping, Linköping University, \\ SE-60174 Norrköping, Sweden
}

(Received 21 March 2012; accepted 15 August 2012; published online 18 September 2012)

\begin{abstract}
Schottky diodes with $\mathrm{Au} / \mathrm{ZnO}$ nanorod (NR)/n-SiC configurations have been fabricated and their interface traps and electrical properties have been investigated by current-voltage (I-V), capacitance-voltage $(\mathrm{C}-\mathrm{V})$, capacitance-frequency (C-f), and conductance-frequency $\left(\mathrm{G}_{\mathrm{p}} / \omega-\omega\right)$ measurements. Detailed and systematic analysis of the frequency-dependent capacitance and conductance measurements was performed to extract the information about the interface trap states. The discrepancy between the high barrier height values obtained from the $\mathrm{I}-\mathrm{V}$ and the $\mathrm{C}-\mathrm{V}$ measurements was also analyzed. The higher capacitance at low frequencies was attributed to excess capacitance as a result of interface states in equilibrium in the $\mathrm{ZnO}$ that can follow the alternating current signal. The energy of the interface states $\left(E_{s s}\right)$ with respect to the valence band at the $\mathrm{ZnO} \mathrm{NR}$ surface was also calculated. The densities of interface states obtained from the conductance and capacitance methods agreed well with each other and this confirm that the observed capacitance and conductance are caused by the same physical processes, i.e., recombination-generation in the interface states. (C) 2012 American Institute of Physics.
\end{abstract}

[http://dx.doi.org/10.1063/1.4752402]

\section{INTRODUCTION}

$\mathrm{ZnO}$ nanostructures have a promising future because of the variety of optical and electrical properties, which make them technologically useful for nanoscale device applications. In recent years, $\mathrm{ZnO}$ nanostructures have been studied with renewed interest with the aim of fabricating devices such as light emitting diodes (LEDs), laser diodes, UV detectors, and solar cells. ${ }^{1}$ The production of high quality $\mathrm{ZnO}$ nanostructure-based homojunctions has proved elusive because of difficulties in the growth of $\mathrm{p}-\mathrm{ZnO}$ materials. ${ }^{2}$ Conversely, the fabrication of heterojunctions and Schottky contacts on $\mathrm{n}-\mathrm{ZnO}$ nanostructures allows the realization of these electronic devices. $\mathrm{SiC}$ is a good candidate for use as a substrate for $\mathrm{ZnO}$ nanostructure growth and further applications. The $\mathrm{SiC}$ and $\mathrm{ZnO}$ have the same wurtzite crystal symmetry and relatively small lattice mismatch $(\sim 5 \%)$. In addition, $\mathrm{SiC}$ has useful properties include excellent electron mobility, high transparency, high break down field, and high thermal conductivity. The original purpose of $\mathrm{SiC}$ material choice was to create $\mathrm{ZnO}$ nanorods based white light emitting diodes that will be stable at high temperatures due the small thermal mismatch, the thermal expansion coefficients of $\mathrm{SiC}$ and $\mathrm{ZnO}$ are $4.3 \times 10^{-6}{ }^{\circ} \mathrm{C}$ and $4.94 \times 10^{-6}{ }^{\circ} \mathrm{C}$, respectively. ${ }^{3}$ Although considerable progress has been made in the fabrication of $\mathrm{ZnO}$ nanostructure-based Schottky diodes, many questions remain about the nature of the electrical transport and the interface states at the metal- $\mathrm{ZnO}$ interface. ${ }^{4} \mathrm{~A}$ stable and good quality rectifying metal contact on the $\mathrm{n}-\mathrm{ZnO}$ surface is crucial for many optoelectronic applications and remains a challenge despite numerous recent investigations. ${ }^{4-7}$ The realization of high quality Schottky contacts on $\mathrm{ZnO}$ nanostructures seems to be difficult because of the interface states, the surface morphology, hydroxide surface contamination, and the subsurface defects, which all play important roles in the electrical properties of these contacts. ${ }^{4}$ In recent years, a number of process methodologies have been developed for the fabrication of reproducible high quality Schottky contacts on $\mathrm{ZnO}$ nanostructures, but controversies remain with regard to the Schottky barrier height and the ideality factor of the ZnO Schottky contacts. ${ }^{2,8-11}$ The deviations in the barrier heights and the ideality factor have been proposed as having been caused by the effects of asymmetric contacts, and the influence of the interfacial layers and/or surface states. ${ }^{9-12}$ In fact, the surfaces of nanostructures should mostly be dominated by surface states because abundant surface states usually exist on the surfaces of these nanostructures. ${ }^{13}$ However, to the best of our knowledge, there have been few reports on Schottky contacts on $\mathrm{ZnO}$ nanostructures, and no previous report has been found that illustrates the influence of the surface states on the barrier potential and rectifying behavior of $\mathrm{ZnO}$ nanostructure Schottky diodes. Capacitance and conductance measurements can provide important information about the interface state energy distribution of the Schottky diodes. In the ideal case, these measurements are frequency independent, but this is often impossible because of the presence of interface states at the metal-semiconductor interface. ${ }^{14,15}$ Schottky barrier heights for bulk $\mathrm{ZnO}$ have been reported in the range of $0.6-0.8 \mathrm{eV}^{2,4}$ In low-dimensional systems, the Schottky barrier height depends not only on the work function of the metal but also on the pinning of the Fermi level by the surface states, image force lowering of the barrier, field penetration and the existence of an interfacial insulating layer; these effects change the absolute current value at low bias values by lowering the Schottky barrier. ${ }^{16}$ Schottky devices can be used to evaluate the different semiconductor parameters, including the carrier density, the Schottky 
barrier height, the carrier density profile, and the band gap discontinuity. The chemical reactions at the metal- $\mathrm{ZnO}$ interface play an important role in Schottky barrier formation. These chemical reactions result in an increase in the density of the oxygen vacancies $\left(\mathrm{V}_{\mathrm{O}}\right)$ close to the interface. The creation of oxygen vacancies becomes energetically more favorable near a metal- $\mathrm{ZnO}$ interface through chemical reactions. This process is too deep to contribute significantly to the unintentional n-type conductivity of $\mathrm{ZnO}$ but is shallow enough to cause the Fermi level pinning at the $\mathrm{ZnO}$ interfaces. ${ }^{17}$ It has been proposed that the oxygen vacancies strongly influence Schottky barrier formation by pinning the $\mathrm{ZnO}$ Fermi level close to the $\mathrm{V}_{\mathrm{O}}(+2,0)$ defect level at $\sim 0.7 \mathrm{eV}$ below the conduction band minimum. ${ }^{17}$ However, good quality Schottky contacts to $\mathrm{ZnO}$ have not been found to degrade in terms of performance over time, so $\mathrm{V}_{\mathrm{O}}$ creation probably occurs simultaneously with the contact formation. An important goal for the fabrication of high quality Schottky contacts should therefore be the minimization of oxygen vacancies near the metal- $\mathrm{ZnO}$ interface. Oxygen plasma can be used to reduce the hydrocarbon and hydroxide accumulation layer on the $\mathrm{ZnO}$ surface, remove the nearsurface defects and donors, and also prevent surface chemisorption of oxygen species. ${ }^{17}$ Mosbacker et al. found that $\mathrm{ZnO}$ exposure to oxygen plasma changes the $\mathrm{Au}$ contacts on $\mathrm{ZnO}$ from ohmic to rectifying contacts, regardless of the surface polarity and the material quality. ${ }^{4} \mathrm{ZnO}$ nanorod (NR) polarity has no influence on the Au Schottky contacts. Several groups have reported on the polarity effect; for example, Allen et al. reported that there is no significant polarity effect on the Au Schottky contacts on $\mathrm{ZnO},{ }^{18}$ while Mosbacker et al. reported that the Au Schottky contacts on $\mathrm{ZnO}$ are almost identical on both the $\mathrm{Zn}$ - and O-polar faces, and that the Au Schottky contacts are independent of both the surface polarity and the material quality. ${ }^{4}$

In this work, we investigate the interface traps and the electrical properties of fabricated ZnO NR-based Schottky diodes by current-voltage (I-V), capacitance-voltage (C-V), capacitance-frequency (C-f), and conductance-frequency $\left(\mathrm{G}_{\mathrm{p}} / \omega-\omega\right)$ measurements. The values of the ideality factor, the barrier height, the density of interface states, and the trap time constant were calculated by using different methods. The density of interface states values obtained from the conductance and capacitance methods confirm that the observed capaci- tance and conductance are caused by the same physical processes of recombination-generation in the interface states.

\section{EXPERIMENTAL DETAILS}

To grow the $\mathrm{ZnO}$ NRs on $\mathrm{n}$-SiC substrates, we used a low temperature chemical growth method. In this method, to improve the $\mathrm{ZnO} \mathrm{NR}$ growth quality, distribution and density, a seed layer is spin-coated and baked for $20 \mathrm{~min}$ at $250{ }^{\circ} \mathrm{C} .{ }^{19}$ Then, zinc nitrate hexahydrate $\left(\mathrm{Zn}\left(\mathrm{NO}_{3}\right)_{2} \cdot 6 \mathrm{H}_{2} \mathrm{O}\right)$ was mixed with hexamethylene tetramine (HMT) $\left(\mathrm{C}_{6} \mathrm{H}_{12} \mathrm{~N}_{4}\right)$ in equal molar concentrations, and the substrates were placed in the resulting solution for $4 \mathrm{~h}$ at $90^{\circ} \mathrm{C}$. After growth, the samples were used to fabricate Schottky diodes. For the ohmic contacts on $\mathrm{n}-\mathrm{SiC}$, we first etched a small portion of $\mathrm{n}$ $\mathrm{SiC}$ which we covered before the growth of the $\mathrm{ZnO}$ NRs and $\mathrm{Al}$ was used to form the ohmic contact to n-SiC. Prior to fabrication of the Schottky contacts, an insulating polymethyl methacrylate layer was deposited between the ZnO NRs. To ensure that no PMMA was on top of the ZnO NRs, oxygen plasma cleaning was performed prior to Au Schottky contact deposition. The Au metal contacts were formed on a group of $\mathrm{ZnO}$ NRs by thermal evaporation at a pressure of $2 \times 10^{-7}$ Torr, and the contacts were formed as circular dots of $1.5 \mathrm{~mm}$ in diameter and $100 \mathrm{~nm}$ in thickness. The device structure was characterized by scanning electron microscopy (SEM), along with I-V, C-V, C-f, and $\mathrm{G}_{\mathrm{p}} / \omega-\omega$ measurements.

\section{RESULTS AND DISCUSSION}

The $\mathrm{ZnO}$ NRs grown were found to be aligned vertically, as shown in the SEM image in Fig. 1, and the inset shows the SEM image after spin coating followed by soft backing. Schottky contacts to $\mathrm{ZnO}$ have been studied for over 40 years, following Mead's pioneering work in the 1960s on vacuum cleaved $\mathrm{ZnO}$ surfaces. ${ }^{20}$ In general, low reactivity metals such as $\mathrm{Au}, \mathrm{Ag}$, and Pd can form Schottky contacts with $\mathrm{n}-\mathrm{ZnO}$, and the ideality factors of these Schottky contacts are considerably higher than unity. ${ }^{21}$ Figure 2(a) shows the typical room temperature (RT) currentvoltage (I-V) characteristics for the $\mathrm{Au} / \mathrm{ZnO}$ NR-based Schottky diode. These I-V characteristics were analyzed using standard thermionic emission theory. According to this theory, the current in such a device can be expressed as

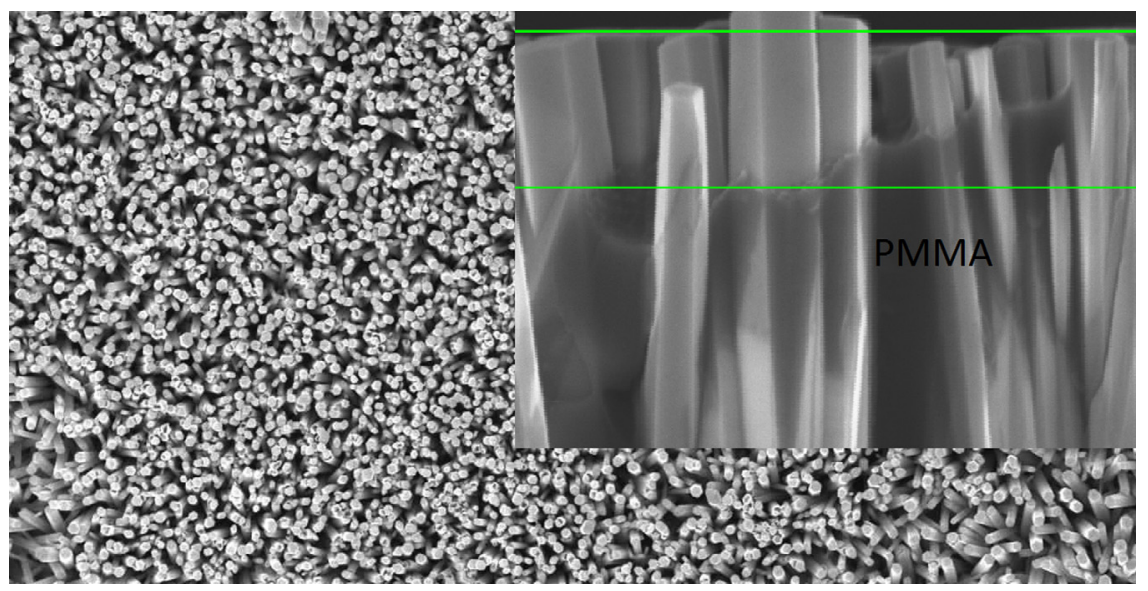

FIG. 1. SEM image of as-grown ZnO NRs on n-SiC substrate. The inset shows the SEM image after spin coating followed by soft backing. 

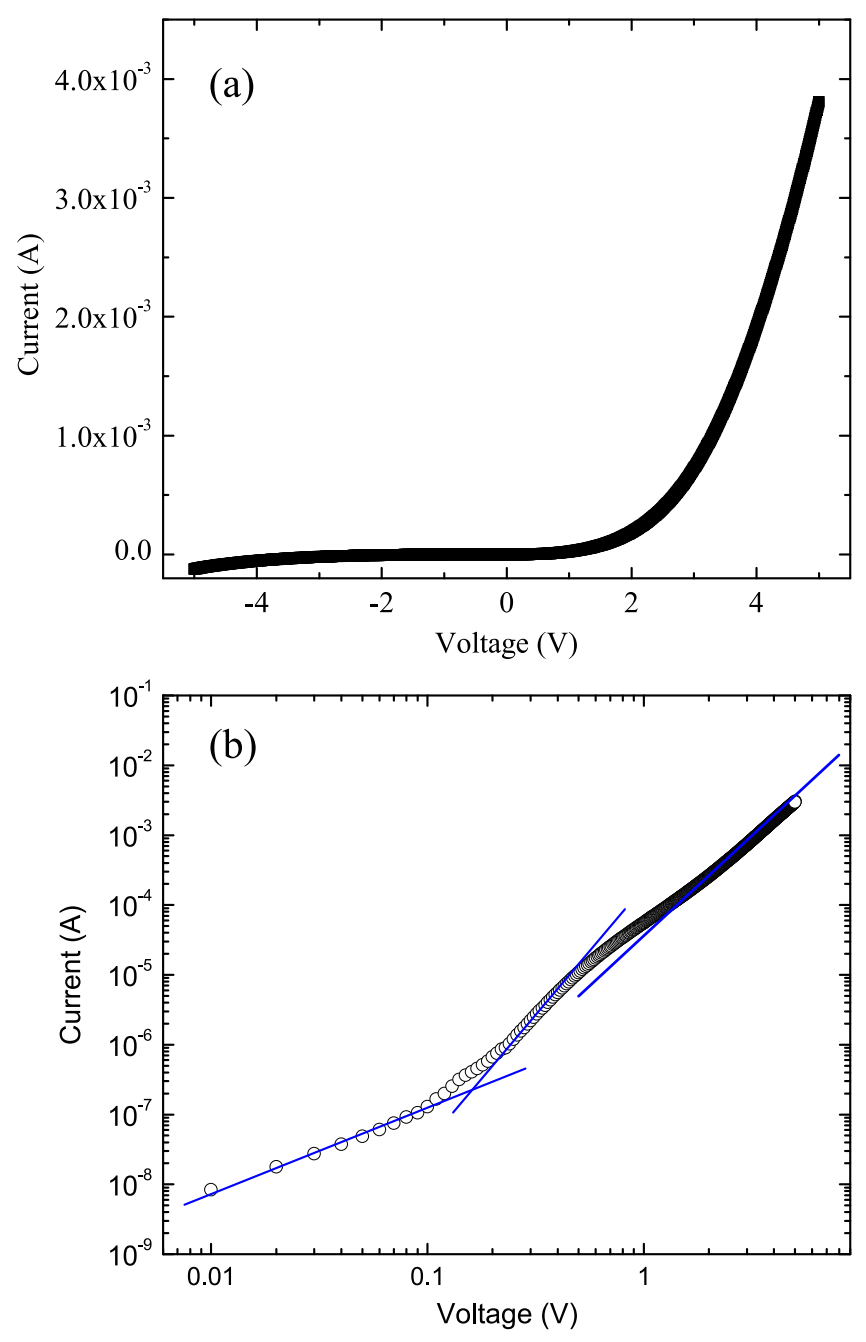

FIG. 2. (a) Typical room temperature current-voltage characteristics for $\mathrm{Au} /$ $\mathrm{ZnO}$ NR-based Schottky diode; and (b) log-log plot for the I-V data of Au/ ZnO NR-based Schottky diode.

$$
I=I_{s}\left[\exp \left(\frac{q\left(V-I R_{s}\right)}{n k T}\right)-1\right]
$$

where $I_{s}$ is the saturation current, $R_{s}$ is the series resistance, $\mathrm{k}$ is the Boltzmann constant, $\mathrm{T}$ is the absolute temperature, $\mathrm{q}$ is the elementary electric charge, $\mathrm{V}$ is the applied voltage, and $n$ is the ideality factor. The saturation current $I_{s}$ is given as

$$
I_{S}=A A^{*} T^{2} \exp \left(-\frac{q \Phi_{b}}{k T}\right)
$$

where $\mathrm{A}$ is the active device area, $\mathrm{A}^{*}$ is the effective Richardson constant, and $\Phi_{\mathrm{b}}$ is the barrier height.

The ideality factor from Eq. (1) was found to be 2.4. The barrier height from Eq. (2) was determined to be $1.0 \mathrm{eV}$. Schottky barrier heights for bulk $\mathrm{ZnO}$ have been reported in the range from $0.6-0.8 \mathrm{eV} .{ }^{2,4} \mathrm{We}$ have a higher barrier height because the microscopic dipole contribution to the built-in potential caused by non-ideal interfacial charge transfer is, in general, accommodated in the $\mathrm{n}-\mathrm{ZnO} / \mathrm{n}-\mathrm{SiC}$ hetero-junction. $^{22}$ The higher ideality factor value obtained is probably caused by the existence of interfacial layers or surface states and also indicates that the transport mechanism is no longer dominated by thermionic emission. To understand the mechanisms which control the junction behavior, the I-V characteristics of the device were studied on a log-log scale.

The logarithmic plot of the I-V characteristics is shown in Fig. 2(b) and it shows three distinct regions, indicating different conduction mechanisms in each region. If deep traps exist at the interface, then the charge transport profile is modified, and these modifications influence the slope of the $\mathrm{I}-\mathrm{V}$ characteristics. The current in region I follows a linear dependence, i.e., $\mathrm{I} \sim \mathrm{V}$. In this region, the injection of carriers from the electrodes into the semiconductor material is reduced considerably because of the low bias voltage. In region II, the current increases exponentially with a relationship of $\mathrm{I} \sim \exp (\mathrm{cV})$. The ideality factor (2.4) is determined in this region and the dominant transport mechanism is recombination-tunneling. Finally, in region III, the current follows a power law $\left(\mathrm{I} \sim \mathrm{V}^{2}\right)$, indicating a space-charge limited current (SCLC) transport mechanism, and this mechanism is controlled by the presence of traps within the band gap of the $\mathrm{ZnO}$ NRs. The SCLC regime occurs when the equilibrium charge concentration is negligible compared with the injected charge concentration and this forms a space charge region near the injecting electrode. ${ }^{23}$

The higher value of the ideality factor must therefore be caused by the existence of surface states. The higher density of surface states would generate upward band bending and would then form a potential barrier on the surface. ${ }^{21}$ The barrier height did not follow the difference in the work function value but was mostly dominated by the surface states or by band bending in the $\mathrm{ZnO}$ itself, as noted by several researchers. $^{21,24-26}$ With a higher density of surface states, the barrier height is controlled by the surface states and dominates the rectifying behaviour. ${ }^{21,26}$ Because of the existence of the surface states, a built-in field can be formed with a direction going from the bulk toward the surface. When a forward bias was applied, with the direction reversed relative to the builtin field, the surface band bending decreased. In contrast, when a reverse bias was applied, the surface band bending increased upward because the direction matches the built-in field. ${ }^{21,25,26}$ The different surface band bending behavior indicated that the intensity of the built-in field was different and this affected the efficiency of the excitons. ${ }^{21} \mathrm{ZnO} \mathrm{NR}$ Schottky contacts have the surface states on the nanorod surface, and this is why the ideality factor and barrier height reported in the literature always had such large deviations.

A typical room temperature $\mathrm{C}-\mathrm{V}$ measurement of a $\mathrm{ZnO}$ Schottky contact is shown in Fig. 3. The carrier concentration and the barrier height were determined from the slope and the intercept of the $1 / \mathrm{C}^{2}$ versus $\mathrm{V}$ plot (Fig. 3) by using the following equations:

$$
\frac{1}{C^{2}}=\frac{2}{q \varepsilon A^{2} N_{D}}\left(V_{b i}-V\right),
$$

where the permittivity $\varepsilon=9.0 \varepsilon_{o}$ for $\mathrm{ZnO}, \mathrm{A}$ is the Schottky contact area, $V_{b i}$ is the built in potential, and $\mathrm{N}_{\mathrm{D}}$ is the carrier concentration, and 


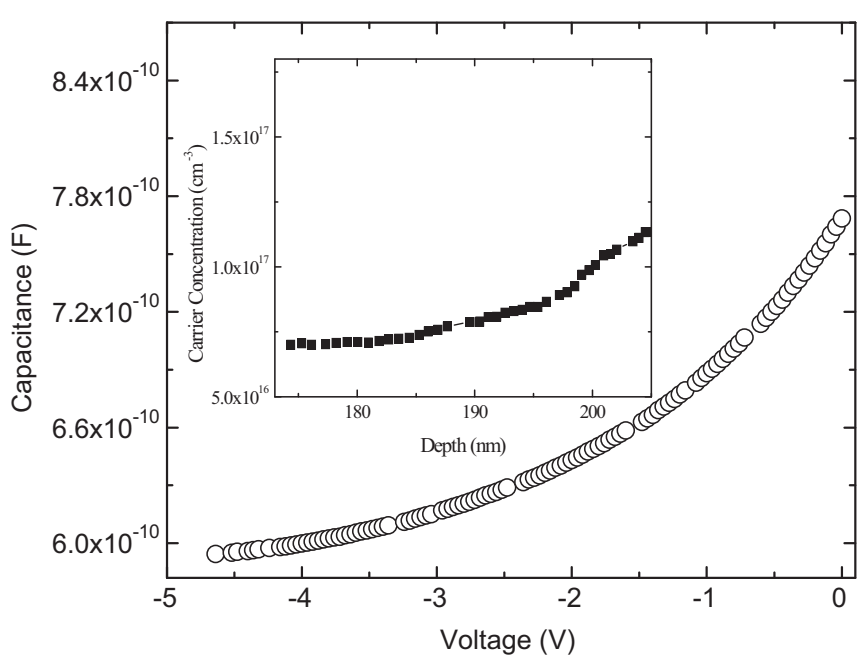

FIG. 3. Typical C-V characteristics of the Au/ZnO NR-based Schottky diode measured at room temperature; the inset shows the depth profile.

$$
\Phi_{B}=V_{b i}+\frac{k T}{q}\left(\frac{N_{C}}{N_{D}}\right),
$$

where $N_{C}=2\left(2 \pi m^{*} k T / h^{2}\right)^{3 / 2}=4.8 \times 10^{18} \mathrm{~cm}^{-3}$ is the conduction band density of states at $\mathrm{T}=300 \mathrm{~K}$. Using Eqs. (3) and (4), the carrier concentration and the barrier height are found to be $7.76 \times 10^{16} \mathrm{~cm}^{-3}$ and $1.26 \mathrm{eV}$, respectively. The barrier height obtained from the $\mathrm{C}-\mathrm{V}$ measurements is higher than that measured using the I-V measurements because of surface defects or because of the effects of the image force and the barrier inhomogeneities. Recently, similar results in a bulk n-ZnO Schottky diode were reported and were attributed to the observation of the surface defects. ${ }^{23}$ The depth profile of the free carrier concentration obtained from the $\mathrm{C}-\mathrm{V}$ measurements is illustrated in the inset of Fig. 3.

Frequency-dependent capacitance and conductance measurements have been successfully used to investigate the interface trap states in ZnO NR Schottky diodes. Capacitance and conductance studies are particularly appropriate for the determination of the effects of the interface trap states. The

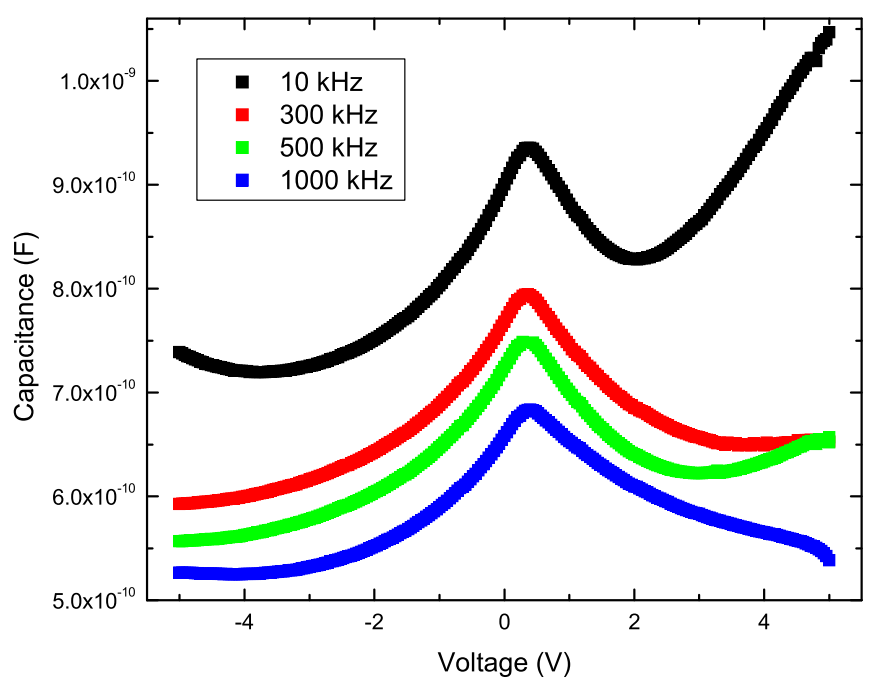

FIG. 4. The $\mathrm{C}-\mathrm{V}$ characteristics of $\mathrm{Au} / \mathrm{ZnO}$ NR-based Schottky diodes at different frequencies. filling and refilling of the trap states cause a measurable change in the capacitance of ZnO Schottky diodes. This loss is caused by the electrons in the conduction band filling the trap states in the band gap, which are at a lower energy, or by the electrons in the trap states being emitted into lower energy valence band states. ${ }^{27}$ It is possible to extract the interface trap density and the times for the trap charging and discharging processes by investigation of the ZnO Schottky diodes at different frequencies. Figure 4 shows the C-V characteristics measured at room temperature at various frequencies. As shown in Fig. 4, the capacitance value decreases for higher frequencies. Normally, the measured capacitance of a Schottky diode depends on the bias voltage and frequency; this dependence is caused by the particular features of the Schottky barrier, the impurity level, the high series resistance, and the interface states and/or interface layer between the Au layer and the $\mathrm{ZnO}$ NRs. At low frequencies, the capacitance is dominated by the depletion capacitance, which is bias-dependent and frequency-independent, but at higher frequencies, the total diode capacitance is affected not only by the depletion capacitance but also by the bulk resistance, which is frequency-dependent and is associated with electron emission from the slowly responding deep impurity levels. ${ }^{23}$ Because of these effects, the capacitance dependence on the bias becomes less pronounced or disappears altogether. The higher capacitance at lower frequencies is because of the excess capacitance that results from the interface states in equilibrium with the $\mathrm{ZnO} \mathrm{NRs}$ that can follow the $\mathrm{AC}$ signal.

Figure 5 shows the room temperature capacitance measured as a function of the frequency (C-f) at zero DC bias; the capacitance is seen to be strongly frequency dependent. The experimental $\mathrm{C}$-f data are compared with the simulated (solid line) results obtained by an exponential fitting procedure, which confirms that the capacitance has an exponential dependence on frequency. This variation in the capacitance is caused by a typical dispersion effect that occurs when the interface states that are in equilibrium with the semiconductor

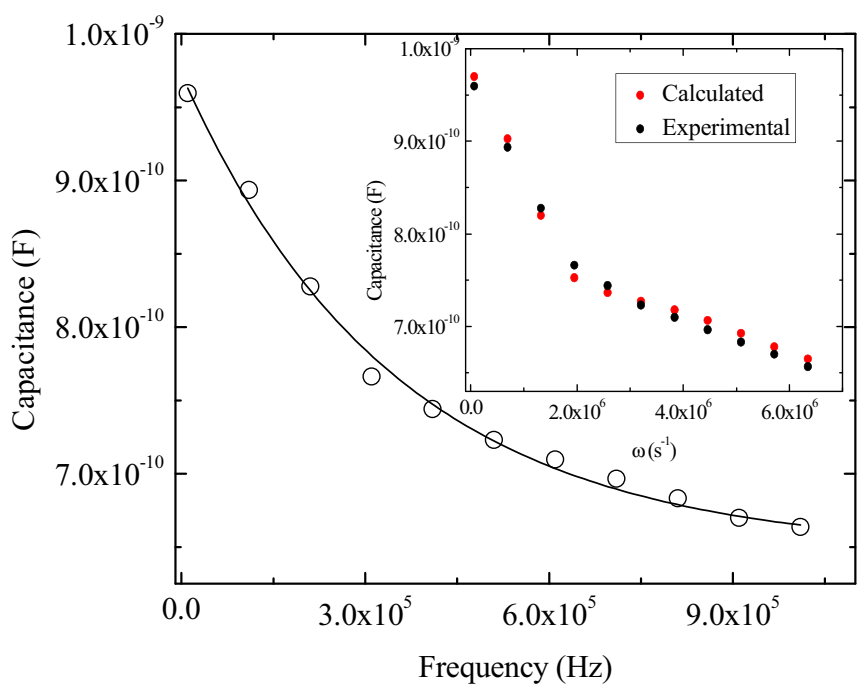

FIG. 5. The $\mathrm{C}-\mathrm{f}$ characteristics of $\mathrm{Au} / \mathrm{ZnO}$ NR-based Schottky diodes at zero bias. The solid line represents the exponential fitting, and the inset shows the $\mathrm{C}_{\mathrm{P}}-\omega$ plot extracted from the measured data. 
do not contribute to the capacitance at sufficiently high frequencies, because the charge at the interface states cannot follow the AC signal. ${ }^{28,29}$ In this case, the capacitance of the Schottky diode is the only space charge capacitance. ${ }^{23}$ The C-f and $G_{p} / \omega-\omega$ measurements give the density distribution of the interface states, the energy distribution of the interface states and the time constants for the rectifying contacts. ${ }^{23}$

The interface state density for the $\mathrm{Au} / \mathrm{ZnO}$ NR-based Schottky diodes can be calculated using the conductance method, in which the parallel conductance is measured as a function of frequency. The parallel capacitance and conductance are expressed as ${ }^{30}$

$$
C_{p}=C_{s}+\frac{C_{i t}}{1+(\omega \tau)^{2}},
$$

where $\omega=2 \pi \mathrm{f}$, and the quantities $\mathrm{C}_{\mathrm{p}}, \mathrm{C}_{\mathrm{s}}, \mathrm{C}_{\mathrm{it}}$, and $\tau$ are the parallel capacitance, the semiconductor capacitance, the interface trap capacitance and the interface trap time constant, respectively. By plotting $C_{P}$ as a function of $\omega$ and fitting the resulting curve to the experimental data, the interface trap density and the interface trap time constant can then be extracted. The $\mathrm{C}_{\mathrm{P}^{-}} \omega$ plot is shown in the inset of Fig. 5 .

The normalized conductance is expressed as ${ }^{30}$

$$
\frac{G_{p}}{\omega}=\frac{q N_{s s}}{2 \omega \tau} \ln \left[1+(\omega \tau)^{2}\right]
$$

where $\mathrm{G}_{\mathrm{p}}, \mathrm{N}_{\mathrm{ss}}$, and $\tau$ are the conductance, the density of interface states and the interface trap time constant, respectively.

The $G_{p} / \omega$ values calculated from the measured capacitance by using Eq. (6) are shown in Fig. 6. The density of interface states and the interface trap time constant can be obtained from the $G_{p} / \omega-\omega$ plot. The peak in the $G_{p} / \omega$ versus $\omega$ plot is caused by the presence of interface traps, which occur at the interfaces of the diodes. For the maximum value of the peak, the first derivative of Eq. (6) was taken and the density of interface states was expressed as

$$
N_{s s}=\frac{2.5}{q}\left(\frac{G_{p}}{\omega}\right)_{\max } .
$$

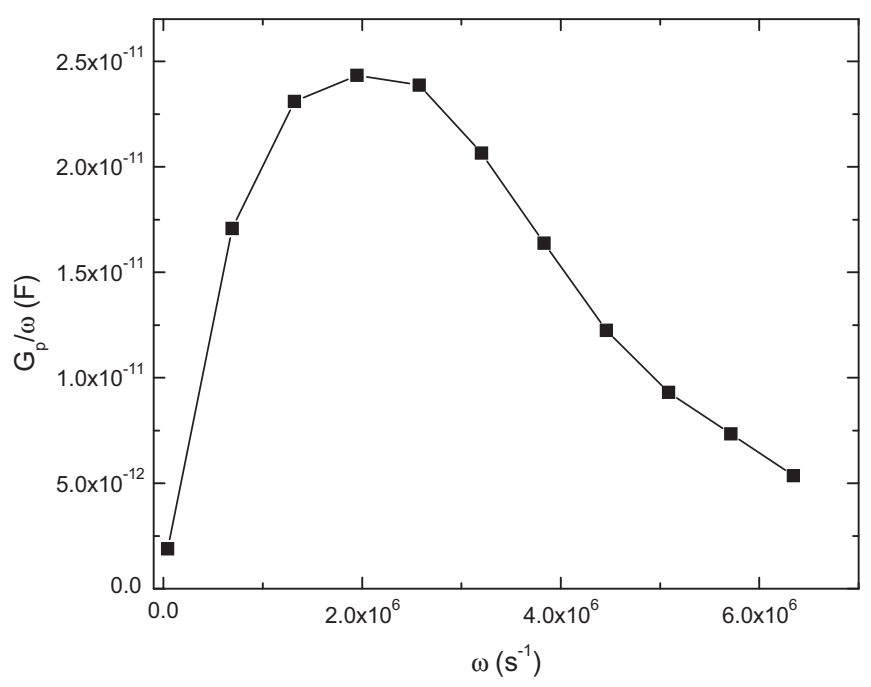

FIG. 6. The $\mathrm{G}_{\mathrm{p}} / \omega$ vs. $\omega$ characteristics extracted from the measured data.
The $\mathrm{G}_{\mathrm{p}} / \omega$ - $\omega$ behavior can be explained by the presence of a continuous distribution of interface state traps. At a given bias, the Fermi level determines the occupancy of these interface trap levels, and a particular interface charge density occurs at the $\mathrm{ZnO} \mathrm{NR}$ surface which determines the time constant of the related interface states. When the AC signal corresponds to this time constant, the peak loss associated with the interface trap levels then occurs. ${ }^{31}$ If the frequency is slightly different to the time constant, then the losses are reduced because either the trap levels do not respond or the response occurs at a different frequency, and therefore a peak loss as a function of frequency is achieved. ${ }^{27,31,32}$ The values of the density of interface states and the interface trap time constant were obtained using the C-f and $G_{p} / \omega$ - $\omega$ measurements and were determined to be $1.9 \times 10^{8} \mathrm{~cm}^{-2} \mathrm{eV}^{-1}$ and $0.5 \mu$ s, respectively.

Only a few groups have reported on the density of interface states in $\mathrm{Au} / \mathrm{ZnO}$ Schottky diodes. We obtained good Schottky contacts on bulk ZnO NRs with a lower interface density when compared with Schottky contacts on $\mathrm{ZnO}$. Yakuphanoglu reported the density of interface states for a $\mathrm{ZnO}$ film to be $1.38 \times 10^{8} \mathrm{~cm}^{-2} \mathrm{eV}^{-1} .33$ Aydoğan et al. reported a value of $17.3 \times 10^{13} \mathrm{~cm}^{-2} \mathrm{eV}^{-1}$ for a $\mathrm{ZnO}$ thin film based Schottky diode. ${ }^{34}$ Faraz et al. reported a range of values of $3.74 \times 10^{11}-7.98 \times 10^{10} \mathrm{~cm}^{-2} \mathrm{eV}^{-1}$ for $\mathrm{ZnO} \mathrm{NR}$ based Schottky diodes. ${ }^{35}$

The energy of the interface states $\mathrm{E}_{\mathrm{ss}}$ with respect to the valence band at the surface of the $\mathrm{ZnO}$ NRs is given by ${ }^{9,36}$

$$
\mathrm{E}_{\mathrm{ss}}-\mathrm{E}_{\mathrm{v}}=\mathrm{E}_{\mathrm{g}}-\varphi_{\mathrm{n}}-\Psi_{\mathrm{s}}
$$

where $\varphi_{\mathrm{n}}=(\mathrm{kT} / \mathrm{q}) \ln \left(\mathrm{N}_{\mathrm{C}} / \mathrm{N}_{\mathrm{D}}\right)$, and $\Psi_{\mathrm{s}}$ is the surface potential.

The total density of interface states is then obtained by integrating $\mathrm{N}_{\mathrm{ss}}$ over the band gap, as shown in Fig 7. $\mathrm{N}_{\mathrm{ss}}$ is obtained by the conductance and capacitance techniques, and the results obtained using these techniques agree well with each other, which confirms that the observed capacitance and conductance are caused by the same physical process, i.e., recombination-generation in the interface states.

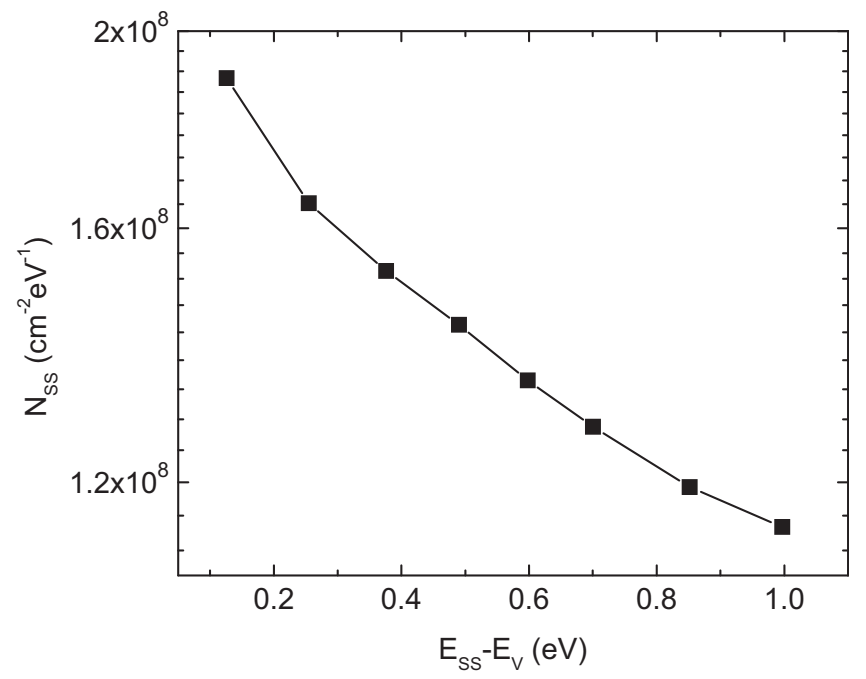

FIG. 7. Energy distribution curve of the interface states calculated from $\mathrm{G}_{\mathrm{p}} /$ $\omega$ vs. $\omega$ measurements. 


\section{CONCLUSIONS}

We have investigated the I-V, C-V, C-f, and $\mathrm{G}_{\mathrm{p}} / \omega-\omega$ characteristics of ZnO NR-based Schottky diodes. The values of the ideality factor, the barrier height, the density of interface states and the trap time constant were calculated by using different methods, and an agreement was observed between the results obtained from these methods. The loglog scale I-V curves exhibited three distinct regions, and the space charge limited current was found to be the dominant transport mechanism in region III. It was found that the capacitance values were dependent on the frequency, and the higher values of capacitance at lower frequencies were attributed to excess capacitance as a result of the interface traps. The capacitance variation is caused by a typical dispersion effect that occurs when the traps are unable to follow the high-frequency voltage modulation and this contributes to the net space charge in the depletion region. The values of the density of interface states and the interface trap time constant that were obtained using the $\mathrm{C}-\mathrm{f}$ and $\mathrm{G}_{\mathrm{p}} /$ $\omega-\omega$ measurements are $1.9 \times 10^{8} \mathrm{~cm}^{-2} \mathrm{eV}^{-1}$ and $0.5 \mu \mathrm{s}$, respectively. The energy of the interface states $E_{s s}$ with respect to the valence band at the surface of the $\mathrm{ZnO}$ NRs was also calculated. The density of interface states values obtained by the conductance and capacitance methods agree well with each other and this confirms that the observed capacitance and conductance are caused by the same physical process, i.e., recombination-generation in the interface states.

${ }^{1}$ M. Willander, O. Nur, Q. X. Zhao, L. L. Yang, M. Lorenz, B. Q. Cao, J. Zúñiga Pérez, C. Czekalla, G. Zimmermann, M. Grundmann, A. Bakin, A. Behrends, M. Al- Suleiman, A. El-Shaer, A. Che Mofor, B. Postels, A. Waag, N. Boukos, A. Travlos, H. S. Kwack, J. Guinard, and D. Le Si Dang, Nanotechnology 20, 332001 (2009).

${ }^{2}$ M. W. Allen, S. M. Durbin, and J. B. Metson, Appl. Phys. Lett. 91(5), 053512 (2007).

${ }^{3}$ N. Bano, I. Hussain, O. Nur, M. Willander, P. Klason, and A. Henry, Semicond. Sci. Technol. 24, 125015 (2009).

${ }^{4}$ H. L. Mosbacker, Y. M. Strzhemechny, and B. D. White, Appl. Phys. Lett. 87(1), 012102 (2005).
${ }^{5}$ S. J. Pearton, D. P. Norton, K. Ip, Y. W. Heo, and T. Steiner, Prog. Mater. Sci. 50, 293 (2005).

${ }^{6}$ A. Salomon, D. Berkovich, and D. Cahen, Appl. Phys. Lett. 82, 1051 (2003).

${ }^{7}$ S.-H. Kim, H.-K. Kim, and T.-Y. Seong, Appl. Phys. Lett. 86, 112101 (2005).

${ }^{8}$ X. Zhang, F. Hai, T. Zhang, C. Jia, X. Sun, L. Ding, and W. Zhang, Microelectron. Eng. 93, 5 (2012).

${ }^{9}$ W. I. Park, G. C. Yi, J. W. Kim, and S. M. Park, Appl. Phys. Lett. 82, 4358 (2003).

${ }^{10}$ O. Harnack, C. Pacholski, H. Weller, A. Yasuda, and J. M. Wessels, Nano Lett. 3, 1097, (2003).

${ }^{11}$ C. S. Lao, J. Liu, P. X. Gao, L. Y. Zhang, D. Davidovic, R. Tummala, and Z. L. Wang, Nano Lett. 2, 263 (2006).

${ }^{12}$ Z. Y. Zhang, C. H. Jin, X. L. Liang, Q. Chen, and L. M. Peng, Appl. Phys. Lett. 88, 073102 (2006).

${ }^{13}$ M. Haase, H. Weller, and A. Henglein, J. Phys. Chem. 92, 482 (1988).

${ }^{14}$ E. H. Rhoderick, Metal-Semiconductor Contacts (Oxford University Press, 1978), pp. 121, 136.

${ }^{15}$ M. Biber, M. Cakar, and A. Turut, J. Mater. Sci.-Mater. Electron. 12, 575 (2001).

${ }^{16}$ H. Kim, H. Kim, and D.-W. Kim, J. Appl. Phys. 108, 074514 (2010).

${ }^{17}$ M. W. Allen and S. M. Durbin, Appl. Phys. Lett. 92, 122110 (2008).

${ }^{18}$ M. W. Allen, M. M. Alkaisi, and S. M. Durbin, Appl. Phys. Lett. 89, 103520 (2006).

${ }^{19}$ M. Vafaee and H. Youzbashizade, Mater. Sci. Forum 553, 252 (2007).

${ }^{20}$ C. A. Mead, Solid-State Electron 9, 1023 (1966).

${ }^{21}$ C. Ke, C. Gang, W. Shujie, Li. Linsong, D. Shuxi, Z. Xingtang, Z. Bingsuo, and Du. Zuliang, New J. Phys. 9, 214 (2007).

${ }^{22}$ J. F. Wager, Thin Solid Films 516, 1755 (2008).

${ }^{23}$ S. Aydogan, M. Saglam, and A. Türüt, Polymer 46, 563 (2005).

${ }^{24}$ J. Bardeen, Phys. Rev. 71, 717 (1947).

${ }^{25}$ S. R. Morrison, The Chemical Physics of Surface (Plenum, NewYork, 1977).

${ }^{26}$ E. H. Rhoderick, Metal-Semiconductor Contacts (Clarendon, Oxford, 1978).

${ }^{27}$ E. H. Nicollian and A. Goetzberger, Bell Syst. Tech. J. 46, 1055 (1967).

${ }^{28}$ J. W. Huang, T. F. Kuech, H. Lu, and I. Bhat, Appl. Phys. Lett. 68, 2392 (1996).

${ }^{29}$ D. Seghier and H. P. Gislason, Appl. Phys. Lett. 88, 6483 (2000).

${ }^{30}$ D. Schroder, Semiconductor Material and Device Characterization, 2nd ed. (Wiley, Toronto, 1998).

${ }^{31}$ M. Çakar, N. Yıldırım, H. Doğan, and A. Türüt Appl. Surf. Sci. 253, 3464 (2007).

${ }^{32}$ M. El Yacoubi, R. Evrard, N. D. Nguyen, and M. Schmeits, Semicond. Sci. Technol. 15, 341 (2000).

${ }^{33}$ F. Yakuphanoglu, Microelectron. Reliab. 51, 2195 (2011).

${ }^{34}$ Ş. Aydoğan, K. Çınar, H. Asıl, C. Coşkun, and A. Türüt, J. Alloys Compd. 476, 913 (2009).

${ }^{35}$ S. M. Faraz, M. Willander, and Q. Wahab, Mater. Sci. Eng. 34, 012006 (2012).

${ }^{36}$ S. Kar and W. E. Dahlke Solid-State Electron. 15, 221 (1972). 